2nd International Conference on Natural Products and Physiologically Active Substances (ICNPAS-2004]

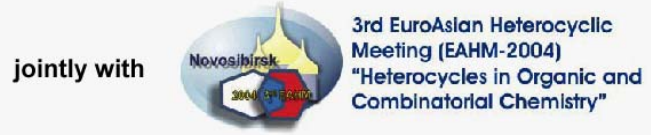

molecules

ISSN 1420-3049

http://www.mdpi.org

\title{
New Multi-1,2,3-Selenadiazole Aromatic Derivatives
}

\section{Mousa Al-Smadi* and Samer Ratrout}

Department of Applied Chemical Sciences, Jordan University of Science and Technology, P.O. Box 3030, Irbid 22110, Jordan

* Author to whom correspondence should be addressed; e-mail: mariam10@just.edu.jo

Received: 23 April 2005 / Published: 30 September 2005

\begin{abstract}
The aromatic polyketones 3a-d are versatile compounds for the synthesis of the multi-1,2,3-selenadiazole aromatic derivatives 1a-d. The preparation starts with the reaction between the multi-bonomethylene farzene-genivatives 2a-d and 4-hydroxyacetophenone to give compounds $3 \mathbf{d}-\mathbf{d}$ which are transformed through the reaction with semicarbazide hydrochloride or ethyl hydrazine carboxylate into the corresponding semicarbazones derivatives $4 \mathbf{4}-\mathbf{d}$ or hydrazones 5a-d. The reaction with selenium dioxide leads to regiospecific ring closure of semicarbazones or hydrazones to give the multi1,2,3-selenadiazole aromatic derivatives in high yield.
\end{abstract}

Keywords: 1,2,3-Selenadiazoles; semicarbazones; aromatic derivatives

\section{Introduction}

Heterocyclic systems with multi-arm 1,2,3-thiadiazoles were recently prepared by Meier et. al [12] and heterocyclic systems containing two 1,2,3-selenadiazole rings were also recently prepared by Reddy et. al [3-4], but multi-arm 1,2,3-selenadiazoles are still unknown. Therefore depending on the previous experience of the principal investigator in synthesizing multi-arm 1,2,3-thiadiazoles, the analogous multi-arm selenadiazoles were prepared following the method that was first reported by Lalezari et. al [5-7], through reaction in the presence of acetic acid of selenium dioxide with $\alpha$ ketomethylene semicarbazones or hydrazones which contain aminocarbonyl or ethoxycarbonyl groups as good leaving groups. NOE measurements showed that the $(E)$-configuration largely predominated around the $\mathrm{CN}$ double bond. 
Selenium containing heterocycles are of increasing interest because of their interesting chemical properties [8-13] and varied biological activities [14-16]. Remarkable differences are known to exist between Se- and S-containing compounds. Due to the larger size of the Se-atom, selenium compounds show an increased polarizability and therefore they are, in general, less stable than the corresponding S-analogues [17-20]. We report herein on our efforts to generate the multi-branched benzene derivatives 1a, $\mathbf{1 b}, \mathbf{1}$ c and $\mathbf{1 d}$, in which the 1,2,3-selenadiazole rings are linked to the central benzene core via phenoxymethylene spacers.

\section{Scheme 1}

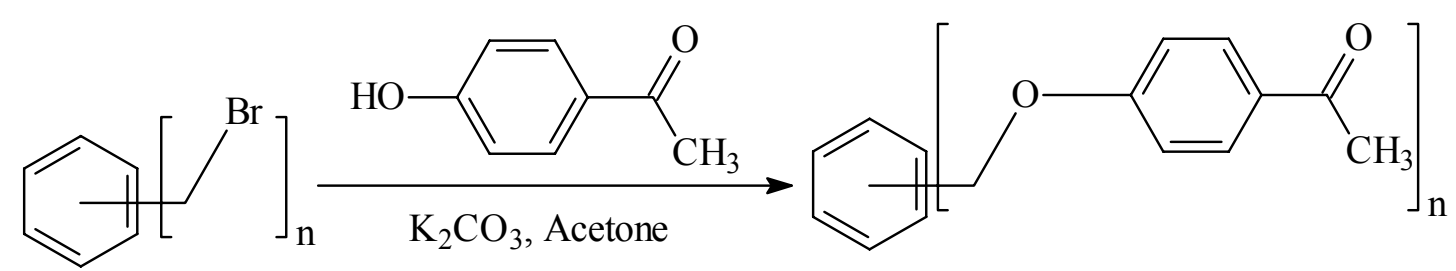

2a-2d

3a-3d

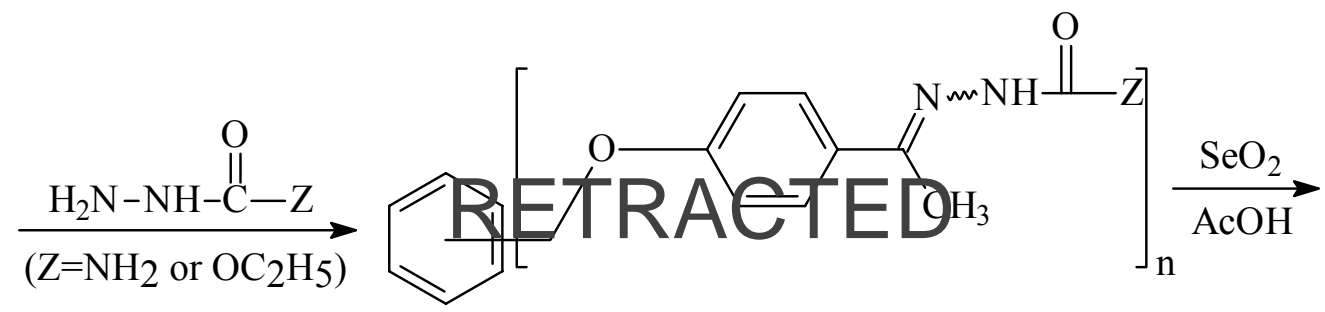

4a-4d

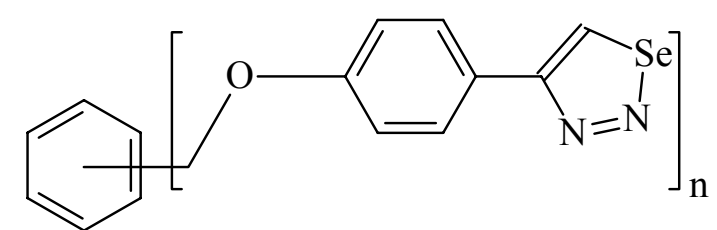

1a-1d

\begin{tabular}{|c|c|}
\hline Series & n \\
\hline a & 6 \\
b & 4 \\
c & 3 \\
d & 2 \\
\hline
\end{tabular}

\section{Results and Discussion}

Our synthetic procedure (see Scheme 1) started from the commercially available bromomethylbenzene derivatives 2a-2d. Multiple substitution with 4-hydroxyacetophenone gave the corresponding polyketones 3a-3d, which were transformed into the target compounds 1a-1d by the reaction of the 
corresponding semicarbazones $\mathbf{4 a - 4 d}$ or ethoxycarbonyl hydrazones $\mathbf{5 a - 5 d}$, essentially as described by Lalezari et. al. [5-7]. The yields of all three steps were optimized, so that the total overall yields for the sequences 2a $\rightarrow$ 1a, $\mathbf{2 b} \rightarrow \mathbf{1 b}, \mathbf{2 c} \rightarrow \mathbf{1 c}$ and $\mathbf{2 d} \rightarrow \mathbf{1 d}$ amounted to $90 \%, 85 \%, 89 \%$ and $85 \%$, respectively (Table 1$)$.

Table 1

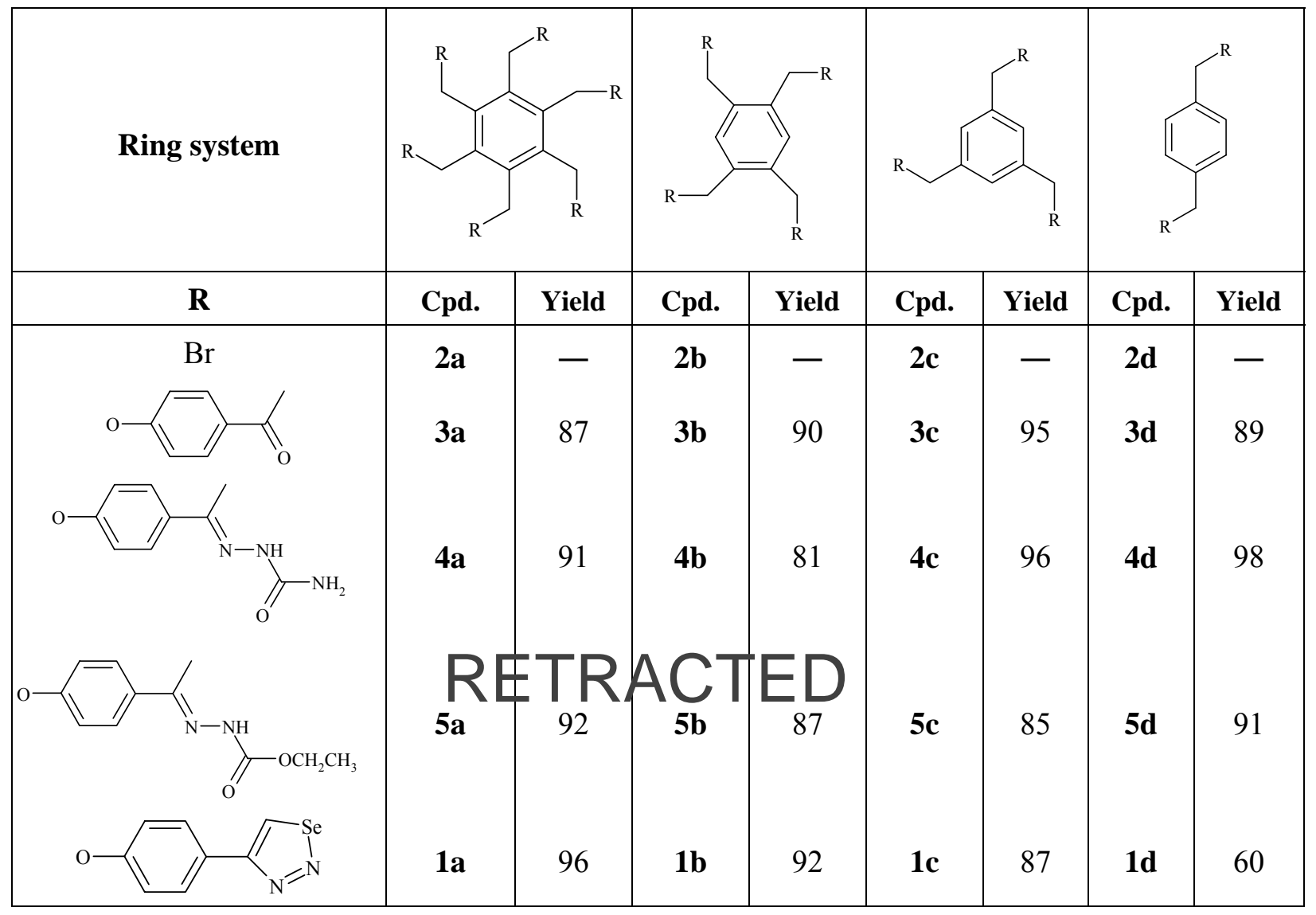

\section{Acknowledgements}

We are grateful to the Deanship of Scientific Research of the Jordan University for Science and Technology for financial support. Also, we thank Prof. H. Meier from Mainz University-Germany for helpful discussions.

\section{Experimental}

\section{General}

The solvents were purified by standard procedures. The melting points (m.p.) were determined on an Electrothermal digital melting point apparatus and are uncorrected. Infrared (IR) spectra of pure substances were recorded as $\mathrm{KBr}$-pellets using a Nicolet 410 FT-IR spectrometer $\left(v\right.$ in $\left.\mathrm{cm}^{-1}\right)$. The ${ }^{1} \mathrm{H}-$ and ${ }^{13} \mathrm{C}$-NMR spectra were recorded on Bruker AM400 and $\mathrm{AC} 200$ spectrometers in $\mathrm{CDCl}_{3}$ or DMSO- $\mathrm{d}_{6}$ using TMS as internal standard. The spectral data are reported in delta $(\delta)$ units relative to 
the TMS reference peak. The mass spectra were recorded using a Finnigan MAT95 field desorption (FD, $5 \mathrm{kV}$ ionizing energy) instrument. The signals are given as $\mathrm{m} / \mathrm{z}$ with the relative intensity between brackets. Elemental analyses were performed in the Analytical Laboratory of the Institute of Organic Chemistry of University of Mainz, Germany. Bromo compounds 2a-2d (1,2,3,4,5,6-hexakis-, 1,2,4,5tetrakis-, 1,3,5-tri- and 1,4-dibromomethylbenzene, respectively), ethyl hydrazine carboxylate, semicarbazide hydrochloride and sodium acetate were obtained from Aldrich.

General Procedure for the Preparation of Multi-Ketones 3a-d [1]

A mixture of 4-hydroxyacetophenone (1 equivalent) and 2a ( 0.14 equivalents), $2 \mathbf{b}$ ( 0.21 equivalents), 2c (0.3 equivalents) or $2 \mathbf{d}$ ( 0.45 equivalents), potassium carbonate ( 1 equivalent) and potassium iodide (in the same equivalent amount as the bromo compound used) plus a few drops of Aliquat 336 were refluxed in dry acetone $(100 \mathrm{~mL})$ for 48 hours. The reaction was followed by TLC (eluent: chloroform) till completion. After cooling, the reaction mixture was diluted with water (50 $\mathrm{mL})$ and extracted with dichloromethane $(3 \times 40 \mathrm{~mL})$. The combined organic layers were dried over magnesium sulphate. The solvent was evaporated under vacuum and the residual solid was washed with diethyl ether. When necessary, a recrystalization from acetone or chloroform was performed.

\section{1-\{4-[2,3,4,5,6-Penta(4-acetylphenoxymethyl)benzyloxy]phenyl\}-1-ethanone (3a).}

Colorless powder $\left(87 \%\right.$ yield), m.p. $234-235^{\circ} \mathrm{C}$; IR: $v 1675,1604,1508,1239,1002,832 \mathrm{~cm}^{-1} ;{ }^{1} \mathrm{H}-$

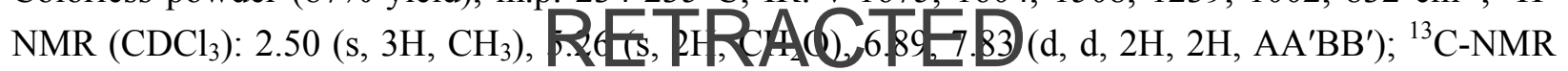
$\left(\mathrm{CDCl}_{3}\right): 26.20\left(\underline{\mathrm{CH}}_{3}\right), 63.60\left(\underline{\mathrm{CH}}_{2} \mathrm{O}\right), 137.70$ (central benzene ring $\left.\mathrm{Cq}\right), 114.21$ and 130.70 (side chain benzene $\mathrm{CH}$ ), 131.30, 161.80 (side chain benzene $\mathrm{Cq}), 196.40(\underline{\mathrm{C}}=\mathrm{O})$; MS: $967\left(\mathrm{M}^{+}, 100\right)$; Anal. \% Calcd. for $\mathrm{C}_{60} \mathrm{H}_{54} \mathrm{O}_{12}$ : C, 74.52; H, 5.63. Found: C, 74.27; H, 5.54.

1-\{4-[2,4,5-Tri(4-acetylphenoxymethyl)benzyloxy]phenyl\}-1-ethanone (3b).

Colorless powder $\left(90 \%\right.$ yield); m.p. $224-226^{\circ} \mathrm{C}$; IR: $v 1665,1590,1502,1250,1170,830 \mathrm{~cm}^{-1}$; ${ }^{1} \mathrm{H}-$ NMR $\left(\mathrm{CDCl}_{3}\right): 2.53\left(\mathrm{~s}, 3 \mathrm{H}, \mathrm{CH}_{3}\right), 5.23\left(\mathrm{~s}, 2 \mathrm{H}, \mathrm{CH}_{2} \mathrm{O}\right), 6.94,7.89$ (d, d, 2H, 2H, AA'B' $\left.\mathrm{BB}^{\prime}\right), 7.65$ (s, benzene ring central $\mathrm{CH}) ;{ }^{13} \mathrm{C}-\mathrm{NMR}\left(\mathrm{CDCl}_{3}\right)$ : $26.30\left(\underline{\mathrm{CH}}_{3}\right), 67.70\left(\underline{\mathrm{CH}}_{2} \mathrm{O}\right), 129.80$ (central benzene ring $\mathrm{CH}$ ), 135.10 (central benzene ring $\mathrm{Cq}$ ), 114.41 and 130.60 (side chain benzene $\mathrm{CH}$ ), 131.10, 162.10 (side chain benzene Cq), $196.50(\underline{\mathrm{C}}=\mathrm{O})$; MS: $670\left(\mathrm{M}^{+}, 100\right)$; Anal. \% Calcd. for $\mathrm{C}_{42} \mathrm{H}_{38} \mathrm{O}_{8}$ : C, 75.21; H, 5.71. Found: C, 75.13; H, 5.59.

1-\{4-[3,5-Di(4-acetylphenoxymethyl)benzyloxy]phenyl\}-1-ethanone (3c).

Pale yellow powder (100\% yield); m.p. 82-83 ${ }^{\circ}$; IR: $v 2910,1670,1591,1500,1250,1172,836 \mathrm{~cm}^{-1}$. ${ }^{1} \mathrm{H}-\mathrm{NMR}\left(\mathrm{DMSO}_{\mathrm{d}}\right.$ ): 2.49 (-s, 3H, $\left.\mathrm{CH}_{3}\right), 5.22$ (s, 2H, $\left.\mathrm{CH}_{2} \mathrm{O}\right), 7.09,7.90$ (d, d, 2H, 2H, AA'BB'), 7.53 (s, benzene ring central $\mathrm{CH}) ;{ }^{13} \mathrm{C}-\mathrm{NMR}\left(\mathrm{DMSO}-\mathrm{d}_{6}\right): 26.30\left(\underline{\mathrm{CH}}_{3}\right), 69.20\left(\underline{\mathrm{CH}}_{2} \mathrm{O}\right), 126.60$ (central benzene ring $\mathrm{CH}$ ), 137.20 (central benzene ring $\mathrm{Cq}$ ), 114.61 and 130.50 (side chain benzene $\mathrm{CH}$ ), 130.10, 162.00 (side chain benzene $\mathrm{Cq}$ ), $196.30(\underline{\mathrm{C}}=\mathrm{O})$; MS: $523\left(\mathrm{M}^{+}, 100\right)$; Anal. \% Calcd. for $\mathrm{C}_{33} \mathrm{H}_{30} \mathrm{O}_{6}$ : C, 75.84; H, 5.79. Found: C, 75.81; H, 5.68. 
1-\{4-[4-Mono-(4-acetylphenoxymethyl) benzyloxy] phenyl\}-1-ethanone (3d).

Colorless crystals $\left(89 \%\right.$ yield); m.p. $181-182^{\circ} \mathrm{C}$; IR: $v$ 1668, 1591, 1239, 996, $823 \mathrm{~cm}^{-1}$; ${ }^{1} \mathrm{H}-\mathrm{NMR}$ $\left(\mathrm{CDCl}_{3}\right): 2.53\left(\mathrm{~s}, 3 \mathrm{H}, \mathrm{CH}_{3}\right), 5.12\left(\mathrm{~s}, 2 \mathrm{H}, \mathrm{CH}_{2} \mathrm{O}\right), 7.00,7.90\left(\mathrm{~d}, \mathrm{~d}, 2 \mathrm{H}, 2 \mathrm{H}, \mathrm{AA}^{\prime} \mathrm{BB}^{\prime}\right), 7.44(\mathrm{~d}$, benzene ring central $\mathrm{CH}) ;{ }^{13} \mathrm{C}-\mathrm{NMR}\left(\mathrm{CDCl}_{3}\right): 26.40\left(\underline{\mathrm{CH}}_{3}\right), 69.70\left(\underline{\mathrm{CH}}_{2} \mathrm{O}\right), 127.80$ (central benzene ring $\left.\mathrm{CH}\right)$, 136.20 (central benzene ring $\mathrm{Cq}$ ), 114.50 and 130.60 (side chain benzene $\mathrm{CH}$ ), 130.60, 162.40 (side chain benzene $\mathrm{Cq}), 196.70(\underline{\mathrm{C}}=\mathrm{O})$; MS: $374\left(\mathrm{M}^{+}, 100\right)$; Anal. \% Calcd. for $\mathrm{C}_{24} \mathrm{H}_{22} \mathrm{O}_{4}$ : C, 76.99; $\mathrm{H}$, 5.92. Found: C, 76.78; H, 5.81.

General procedure for the preparation of multiple semicarbazones 4a-d

A mixture of semicarbazide hydrochloride ( 1 equivalent) and sodium acetate ( 1 equivalent) was dissolved in absolute ethanol $(40 \mathrm{~mL})$. The mixture was heated for $15 \mathrm{~min}$ under reflux, then filtered while hot to remove precipitated sodium chloride. The filtrate was mixed with ketone 3a $(0.14$ equivalents), ketone $\mathbf{3 b}$ (0.21 equivalents), ketone $3 \mathbf{c}$ ( 0.30 equivalents) or ketone $3 \mathbf{d}$ (0.45 equivalents), respectively. The reaction mixture was heated to reflux then two drops of concentrated hydrochloric acid were added. The mixture was heated under reflux for overnight with continuously removal of generated water. After that the solvent was removed under vacuum and the residue was washed with diethyl ether.

1-\{4-[2,3,4,5,6-Penta(4-acetylphenoxymethyl) benzyloxy]phenyl\}-1-ethanone- $N$-aminocarbonyl semicarbazone (4a).

RETRACTED

White powder (91\% yield), m.p. $300^{\circ} \mathrm{C}$ (dec.); IR: $v 3417,3212,1687,1597,1431,1239,1014,829$ $\mathrm{cm}^{-1}$; ${ }^{1} \mathrm{H}-\mathrm{NMR}\left(\mathrm{DMSO}_{6}\right)$ : $\left.2.08\left(\mathrm{~s}, 3 \mathrm{H}, \mathrm{CH}_{3}-\mathrm{C}=\mathrm{N}\right)\right), 5.37\left(\mathrm{~s}, 2 \mathrm{H}, \mathrm{CH}_{2} \mathrm{O}\right), 6.41\left(\mathrm{~s}, 2 \mathrm{H}, \mathrm{NH}_{2}\right), 6.93$, $7.70\left(\mathrm{~d}, \mathrm{~d}, 2 \mathrm{H}, 2 \mathrm{H}, \mathrm{AA}^{\prime} \mathrm{BB}^{\prime}\right), 9.18(\mathrm{~s}, 1 \mathrm{H}, \mathrm{N}-\mathrm{H}) ;{ }^{13} \mathrm{C}-\mathrm{NMR}\left(\mathrm{DMSO}-\mathrm{d}_{6}\right): 13.29\left(\underline{\mathrm{CH}}_{3}-\mathrm{C}=\mathrm{N}\right), 63.90$ $\left(\underline{\mathrm{CH}}_{2} \mathrm{O}\right.$ ), 137.60 (central benzene ring $\mathrm{Cq}$ ), 114.30 and 127.50 (side chain benzene $\mathrm{CH}$ ), 130.40, 159.60 (side chain benzene $\mathrm{Cq}), 143.90(\underline{\mathrm{C}}=\mathrm{N}), 157.40(\underline{\mathrm{C}}=\mathrm{O})$; MS: $1309.4\left(\mathrm{M}^{+}, 100\right)$; Anal. \% Calcd. for $\mathrm{C}_{66} \mathrm{H}_{72} \mathrm{~N}_{18} \mathrm{O}_{12}$ : C, 60.54; H, 5.54; N, 19.25, Found: C, 60.35; H, 5.54; N, 19.30 .

1-\{4-[2,4,5-Tri(4-acetylphenoxymethyl)benzyloxy]phenyl\}-1-ethanone- $N$-aminocarbonylsemicarbazone (4b).

White powder (81\% yield); m.p. $250^{\circ} \mathrm{C}$ (dec.); IR: $v 3429,3180,1670,1599,1418,1258,836 \mathrm{~cm}^{-1}$; ${ }^{1} \mathrm{H}-\mathrm{NMR}\left(\mathrm{DMSO}_{\mathrm{d}}\right)$ ) $\left.2.12\left(\mathrm{~s}, 3 \mathrm{H}, \mathrm{CH}_{3}-\mathrm{C}=\mathrm{N}\right)\right), 5.34\left(\mathrm{~s}, 2 \mathrm{H}, \mathrm{CH}_{2} \mathrm{O}\right), 5.99\left(\mathrm{~s}, 2 \mathrm{H}, \mathrm{NH}_{2}\right), 7.06,7.86(\mathrm{~d}$, $\left.\mathrm{d}, 2 \mathrm{H}, 2 \mathrm{H}, \mathrm{AA}^{\prime} \mathrm{BB}^{\prime}\right), 7.71$ (s, central benzene ring $\mathrm{CH}$ ), 9.19 (s, 1H, N-H); ${ }^{13} \mathrm{C}-\mathrm{NMR}\left(\mathrm{DMSO}-\mathrm{d}_{6}\right)$ : $13.38\left(\underline{\mathrm{CH}}_{3}-\mathrm{C}=\mathrm{N}\right), 67.08\left(\underline{\mathrm{CH}}_{2} \mathrm{O}\right), 129.10$ (central benzene ring $\left.\mathrm{CH}\right), 134.80$ (central benzene ring $\mathrm{Cq}$ ), 114.70 and 127.50 (side chain benzene $\mathrm{CH}$ ), 130.50, 159.70 (side chain benzene $\mathrm{Cq}$ ), $144.00(\underline{\mathrm{C}}=\mathrm{N})$, $157.50(\underline{\mathrm{C}}=\mathrm{O})$; MS: $899\left(\mathrm{M}^{+}, 100\right)$; Anal. \% Calcd. for $\mathrm{C}_{46} \mathrm{H}_{50} \mathrm{~N}_{12} \mathrm{O}_{8}: \mathrm{C}, 62.33 ; \mathrm{H}, 5.67 ; \mathrm{N}, 18.17$, Found: C, 62.29; H, 5.61; N, 18.05 . 
1-\{4-[3,5-Di(4-acetylphenoxymethyl)benzyloxy]phenyl\}-1-ethanone-N-aminocarbonyl semicarbazone (4c).

Pale yellow powder (100\% yield); m.p. $240^{\circ} \mathrm{C}$ (dec.); IR: v 3417, 3250, 1681, 1579, 1508, 1470, 1226, $829 \mathrm{~cm}^{-1}$; $\left.{ }^{1} \mathrm{H}-\mathrm{NMR}\left(\mathrm{DMSO}_{6}\right): 2.13\left(\mathrm{~s}, 3 \mathrm{H}, \mathrm{CH}_{3}-\mathrm{C}=\mathrm{N}\right)\right), 5.15\left(\mathrm{~s}, 2 \mathrm{H}, \mathrm{CH}_{2} \mathrm{O}\right), 6.55\left(\mathrm{~s}, 2 \mathrm{H}, \mathrm{NH}_{2}\right), 6.98$, $7.76\left(\mathrm{~d}, \mathrm{~d}, 2 \mathrm{H}, 2 \mathrm{H}, \mathrm{AA}^{\prime} \mathrm{BB}^{\prime}\right), 7.49$ (s, central benzene ring $\left.\mathrm{CH}\right), 9.25$ (s, 1H, N-H); ${ }^{13} \mathrm{C}-\mathrm{NMR}$ (DMSO$\left.\mathrm{d}_{6}\right): 13.38\left(\underline{\mathrm{CH}}_{3}-\mathrm{C}=\mathrm{N}\right), 69.15\left(\underline{\mathrm{CH}}_{2} \mathrm{O}\right), 126.44$ (central benzene ring $\left.\mathrm{CH}\right), 137.76$ (central benzene ring $\mathrm{Cq}$ ), 114.54 and 127.50 (side chain benzene $\mathrm{CH}$ ), 131.20, 158.73 (side chain benzene $\mathrm{Cq}$ ), 144.11 $(\underline{\mathrm{C}}=\mathrm{N}), 157.90(\underline{\mathrm{C}}=\mathrm{O})$; MS: $693.77\left(\mathrm{M}^{+}, 100\right)$; Anal. \% Calcd. for $\mathrm{C}_{36} \mathrm{H}_{39} \mathrm{~N}_{9} \mathrm{O}_{6}: \mathrm{C}, 61.46 ; \mathrm{H}, 5.61 ; \mathrm{N}$, 18.70, Found: C, 63.32; H, 5.51; N, 18.63.

1-\{4-[4-(4-acetylphenoxymethyl)benzyloxy]phenyl\}-1-ethanone-N-aminocarbonyl semicarbazone (4d).

White powder (100\% yield); m.p. $300^{\circ} \mathrm{C}$ (dec.); IR: $v 3417,3212,1681,1604,1502,1418,1239$, 1014, $829 \mathrm{~cm}^{-1}$; $\left.{ }^{1} \mathrm{H}-\mathrm{NMR}\left(\mathrm{DMSO}_{\mathrm{d}}\right): 2.12\left(\mathrm{~s}, 3 \mathrm{H}, \mathrm{CH}_{3}-\mathrm{C}=\mathrm{N}\right)\right), 5.20\left(\mathrm{~s}, 2 \mathrm{H}, \mathrm{CH}_{2} \mathrm{O}\right), 5.90(\mathrm{~s}, 2 \mathrm{H}$, $\left.\mathrm{NH}_{2}\right), 7.08,7.89\left(\mathrm{~d}, \mathrm{~d}, 2 \mathrm{H}, 2 \mathrm{H}, \mathrm{AA}^{\prime} \mathrm{BB}^{\prime}\right), 7.45$ (d, central benzene ring $\left.\mathrm{CH}\right), 9.21(\mathrm{~s}, 1 \mathrm{H}, \mathrm{N}-\mathrm{H})$; ${ }^{13} \mathrm{C}-$ NMR (DMSO-d $\left.)_{6}\right): 13.30\left(\underline{\mathrm{CH}}_{3}-\mathrm{C}=\mathrm{N}\right), 69.30\left(\underline{\mathrm{CH}}_{2} \mathrm{O}\right), 127.80$ (central benzene ring $\left.\mathrm{CH}\right), 136.41$ (central benzene ring $\mathrm{Cq}$ ), 114.50 and 128.00 (side chain benzene $\mathrm{CH}$ ), 131.17, 159.70 (side chain benzene $\mathrm{Cq}), 143.00(\underline{\mathrm{C}}=\mathrm{N}), 158.70(\underline{\mathrm{C}}=\mathrm{O})$; MS: $488.5\left(\mathrm{M}^{+}, 100\right)$; Anal. \% Calcd. for $\mathrm{C}_{26} \mathrm{H}_{28} \mathrm{~N}_{6} \mathrm{O}_{4}$ : $\mathrm{C}$, 63.92; H, 5.78; N, 17.20, Found: C, 63.71; H, 5.72; N, 17.15 .

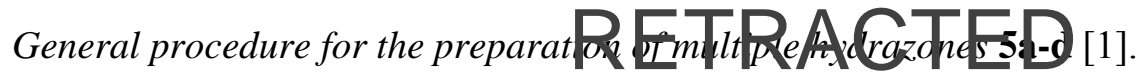

A solution of di- or tri- or tetra- or hexaketone 3a-d (1 equivalent), a few drops of concentrated hydrochloric acid and 6, 9, 12 or 18 equivalents of ethyl hydrazinecarboxylate in dry chloroform (50 $\mathrm{mL}$ ) was heated under reflux overnight with continuously removal of generated water. The solution was concentrated and the residue was washed with diethyl ether and chloroform.

1-\{4-[2,3,4,5,6-Penta(4-acetylphenoxymethyl)benzyloxy]phenyl\}-1-ethanone- $N$-ethoxycarbonyl hydrazone (5a).

White solid powder (92\% yield); m.p. $293^{\circ} \mathrm{C}$ (dec.); IR: v 3231, 1719, 1610, 1502, 1233, 1047, 829 $\mathrm{cm}^{-1}$; ${ }^{1} \mathrm{H}-\mathrm{NMR}$ (DMSO-d $)$ ): 1.23 (t, 3H, $\underline{\mathrm{CH}}_{3} \mathrm{CH}_{2}$ ), 2.12 (s, 3H, $\left.\mathrm{CH}_{3}-\mathrm{C}=\mathrm{N}\right)$ ), 4.13 (q, 2H, $\mathrm{CH}_{3} \underline{\mathrm{CH}}_{2}$ ), 5.29 (s, 2H, $\left.\mathrm{CH}_{2} \mathrm{O}\right), 6.95,7.58\left(\mathrm{~d}, \mathrm{~d}, 2 \mathrm{H}, 2 \mathrm{H}, \mathrm{AA}^{\prime} \mathrm{BB}^{\prime}\right), 9.96(\mathrm{~s}, 1 \mathrm{H}, \mathrm{N}-\mathrm{H}) ;{ }^{13} \mathrm{C}-\mathrm{NMR}\left(\mathrm{DMSO}-\mathrm{d}_{6}\right)$ : $14.23\left(\underline{\mathrm{CH}}_{3} \mathrm{CH}_{2}\right), 15.01\left(\underline{\mathrm{CH}}_{3}-\mathrm{C}=\mathrm{N}\right), 60.95\left(\mathrm{CH}_{3} \underline{\mathrm{CH}}_{2} \mathrm{O}\right), 64.36\left(\underline{\mathrm{CH}}_{2} \mathrm{O}-\mathrm{ph}\right), 138.09$ (central benzene ring $\mathrm{Cq}$ ), 114.86 and 127.94 (side chain benzene $\mathrm{CH}$ ), 131.93, 159.41 (side chain benzene $\mathrm{Cq}$ ), 149.25 $(\underline{\mathrm{C}}=\mathrm{N}), 154.76\left(\underline{\mathrm{CO}_{2}}\right)$; MS: $1484\left(\mathrm{M}^{+}, 100\right)$; Anal. \% Calcd. for $\mathrm{C}_{78} \mathrm{H}_{90} \mathrm{~N}_{12} \mathrm{O}_{18}: \mathrm{C}, 63.15 ; \mathrm{H}, 6.11 ; \mathrm{N}$, 11.33, Found: C, 62.79; H, 6.27; N, 11.44. 
1-\{4-[2,4,5-Tri-(4-acetylphenoxymethyl)benzyloxy]phenyl\}-1-ethanone-N-ethoxycarbonyl hydrazone (5b).

White powder (87\% yield), m.p. $211-213^{\circ} \mathrm{C}$; IR: $v 3200,3045,1700,1596,1492,1235,1040,828$ $\mathrm{cm}^{-1}$; ${ }^{1} \mathrm{H}-\mathrm{NMR}\left(\mathrm{DMSO}-\mathrm{d}_{6}\right): 1.24$ (t, $\left.3 \mathrm{H}, \underline{\mathrm{CH}}_{3} \mathrm{CH}_{2}\right), 2.16$ (s, 3H, $\left.\mathrm{CH}_{3}-\mathrm{C}=\mathrm{N}\right)$ ), $4.16\left(\mathrm{q}, 2 \mathrm{H}, \mathrm{CH}_{3} \underline{\mathrm{CH}}_{2}\right)$, $5.27\left(\mathrm{~s}, 2 \mathrm{H}, \mathrm{CH}_{2} \mathrm{O}\right), 7.03,7.65\left(\mathrm{~d}, \mathrm{~d}, 2 \mathrm{H}, 2 \mathrm{H}, \mathrm{AA}^{\prime} \mathrm{BB}^{\prime}\right), 7.71(\mathrm{~s}$, central benzene ring $\mathrm{CH}), 9.98(\mathrm{~s}, 1 \mathrm{H}$, $\mathrm{N}-\mathrm{H}) ;{ }^{13} \mathrm{C}-\mathrm{NMR}\left(\mathrm{DMSO}-\mathrm{d}_{6}\right): 14.28\left(\underline{\mathrm{CH}}_{3} \mathrm{CH}_{2}\right), 15.19\left(\underline{\mathrm{CH}}_{3}-\mathrm{C}=\mathrm{N}\right), 60.98\left(\mathrm{CH}_{3} \underline{C H}_{2} \mathrm{O}\right), 67.40\left(\underline{\mathrm{CH}}_{2} \mathrm{O}-\right.$ ph), 129.37 (central benzene ring $\mathrm{CH}$ ), 135.43 (central benzene ring $\mathrm{Cq}$ ), 115.08 and 128.00 (side chain benzene $\mathrm{CH}), 131.76,159.33$ (side chain benzene $\mathrm{Cq}), 148.70(\underline{\mathrm{C}}=\mathrm{N}), 154.80\left(\underline{\mathrm{CO}_{2}}\right)$; $\mathrm{MS}$ : 1015 $\left(\mathrm{M}^{+}, 100\right)$; Anal. \% Calcd. for $\mathrm{C}_{54} \mathrm{H}_{62} \mathrm{~N}_{8} \mathrm{O}_{12}$ : C, 63.89; H, 6.16; N, 11.04, Found: $\mathrm{C}, 63.58 ; \mathrm{H}, 6.09$; $\mathrm{N}$, 10.97 .

1-\{4-[3,5-Di(4-acetylphenoxymethyl)benzyloxy]phenyl\}-1-ethanone-N-ethoxycarbonyl hydrazone (5c).

Pale yellow powder (85\% yield), m.p. 202-204 C; IR: $v$ 3200, 3035, 1705, 1600, 1503, 1238, 1040, $830 \mathrm{~cm}^{-1} ;{ }^{1} \mathrm{H}-\mathrm{NMR}\left(\mathrm{DMSO}_{6}\right): \delta 1.24\left(\mathrm{t}, 3 \mathrm{H}, \mathrm{CH}_{3} \mathrm{CH}_{2}\right), 2.17\left(\mathrm{~s}, 3 \mathrm{H}, \mathrm{CH}_{3}-\mathrm{C}=\mathrm{N}\right)$ ), $4.15(\mathrm{q}, 2 \mathrm{H}$, $\left.\mathrm{CH}_{3} \mathrm{CH}_{2}\right), 5.18$ (s, 2H, $\left.\mathrm{CH}_{2} \mathrm{O}\right), 7.03,7.67$ (d, d, 2H, 2H, $\left.\mathrm{AA}^{\prime} \mathrm{BB}^{\prime}\right), 7.52$ (s, central benzene ring $\mathrm{CH}$ ), $9.99(\mathrm{~s}, 1 \mathrm{H}, \mathrm{N}-\mathrm{H}) ;{ }^{13} \mathrm{C}-\mathrm{NMR}\left(\mathrm{DMSO}-\mathrm{d}_{6}\right): \delta 14.30\left(\underline{\mathrm{CH}}_{3} \mathrm{CH}_{2}\right), 15.20\left(\underline{\mathrm{CH}}_{3}-\mathrm{C}=\mathrm{N}\right), 60.90\left(\mathrm{CH}_{3} \underline{\mathrm{CH}}_{2} \mathrm{O}\right)$, $69.60\left(\mathrm{CH}_{2} \mathrm{O}-\mathrm{ph}\right), 126.90$ (central benzene ring $\mathrm{CH}$ ), 138.06 (central benzene ring $\mathrm{Cq}$ ), 115.10 and 128.00 (side chain benzene $\mathrm{CH}$ ), 131.60, 159.50 (side chain benzene $\mathrm{Cq}), 148.70(\underline{\mathrm{C}}=\mathrm{N}), 154.20$ $\left(\underline{\mathrm{CO}_{2}}\right)$; MS: $781\left(\mathrm{M}^{+}, 100\right)$; Anal. \% Calcd. for $\mathrm{C}_{42} \mathrm{H}_{48} \mathrm{~N}_{6} \mathrm{O}_{9}: \mathrm{C}, 64.60 ; \mathrm{H}, 6.20 ; \mathrm{N}, 10.76$, Found: $\mathrm{C}$, 64.48; H, 6.13; N, 10.50 .

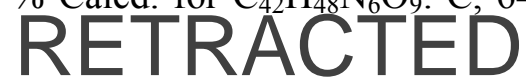

1-\{4-[4-Mono(4-acetylphenoxymethyl)benzyloxy]phenyl\}-1-ethanone-N-ethoxycarbonyl hydrazone (5d).

White powder (91\% yield); m.p. $259-260{ }^{\circ} \mathrm{C}$; IR: $v$ 3205, 3035, 1705, 1600, 1500, 1230, $822 \mathrm{~cm}^{-1}$; ${ }^{1} \mathrm{H}-$ NMR (DMSO-d $\left.\left.)_{6}\right): \delta 1.22\left(\mathrm{t}, 3 \mathrm{H}, \underline{\mathrm{CH}}_{3} \mathrm{CH}_{2}\right), 2.15\left(\mathrm{~s}, 3 \mathrm{H}, \mathrm{CH}_{3}-\mathrm{C}=\mathrm{N}\right)\right), 4.16\left(\mathrm{q}, 2 \mathrm{H}, \mathrm{CH}_{3} \mathrm{CH}_{2}\right), 5.10(\mathrm{~s}$, $\left.2 \mathrm{H}, \mathrm{CH}_{2} \mathrm{O}\right), 6.99,7.87\left(\mathrm{~d}, \mathrm{~d}, 2 \mathrm{H}, 2 \mathrm{H}, \mathrm{AA}^{\prime} \mathrm{BB}^{\prime}\right), 7.43$ (d, central benzene ring $\left.\mathrm{CH}\right), 9.99(\mathrm{~s}, 1 \mathrm{H}, \mathrm{N}-\mathrm{H})$; ${ }^{13} \mathrm{C}-\mathrm{NMR}\left(\mathrm{DMSO}-\mathrm{d}_{6}\right): \delta 13.60\left(\underline{\mathrm{CH}}_{3} \mathrm{CH}_{2}\right), 14.70\left(\underline{\mathrm{CH}}_{3}-\mathrm{C}=\mathrm{N}\right), 60.80\left(\mathrm{CH}_{3} \underline{\mathrm{CH}}_{2} \mathrm{O}\right), 67.80\left(\underline{\mathrm{CH}}_{2} \mathrm{O}-\mathrm{Ph}\right)$, 127.60 (central benzene ring $\mathrm{CH}$ ), 136.30 (central benzene ring $\mathrm{Cq}$ ), 114.50 and 127.60 (side chain benzene $\mathrm{CH}), 130.50,160.10$ (side chain benzene $\mathrm{Cq}), 148.81(\underline{\mathrm{C}}=\mathrm{N}), 154.30\left(\underline{\mathrm{CO}}_{2}\right)$; MS: $547\left(\mathrm{M}^{+}\right.$, 100); Anal. \% Calcd. for $\mathrm{C}_{30} \mathrm{H}_{34} \mathrm{~N}_{4} \mathrm{O}_{6}$ : C, 65.92; H, 6.27; N, 10.25, Found: C, 65.99; H, 6.18; N, 10.15.

\section{General procedure for preparation of multiple 1,2,3-selenadiazoles 1a-d}

Hydrazone $5 \mathbf{a}(0.47 \mathrm{mmol})$ or $5 \mathbf{b}(0.59 \mathrm{mmol})$ or $\mathbf{5 c}(0.9 \mathrm{mmol})$ or $5 \mathbf{d}(0.27 \mathrm{mmol})$ was dissolved in glacial acetic acid $(30 \mathrm{~mL})$ with vigorous stirring and gentle heating to $40-45^{\circ} \mathrm{C}$. The solution was treated with selenium dioxide powder $(8.46 \mathrm{mmol}, 7.08 \mathrm{mmol}, 8.1 \mathrm{mmol}$ or $0.81 \mathrm{mmol}$, respectively) and the mixture was kept under gentle heating with vigorous stirring. After $2 \mathrm{~min}$, the color of the mixture becomes red. Monitoring of the reaction by TLC showed that the reaction was complete in two days. The mixture was filtered and the filtrate was poured into ice water and extracted with chloroform $(3 \times 50 \mathrm{~mL})$. The combined organic layers were washed with saturated sodium hydrogen 
carbonate solution and dried using magnesium sulphate. The solvent was removed under vacuum. The crude product was purified by chromatography using methanol or ethyl acetate as eluents, followed by recrystallization from chloroform/hexane.

4-(4-\{2,3,4,5,6-Penta[4-(1,2,3-selenadiazole-4-yl)phenoxymethyl]benzyloxy\}phenyl)-1,2,3selenadiazole (1a).

Yellow-orange solid (100\%); m.p. 77-78 C; IR: v 3096, 2975, 1739, 1611, 1527, 1469, 1220, 989, 841 $\mathrm{cm}^{-1}$; ${ }^{1} \mathrm{H}-\mathrm{NMR}\left(\mathrm{CDCl}_{3}\right): 5.27$ (s, 2H, $\left.\mathrm{CH}_{2} \mathrm{O}\right), 7.06,7.17$ (d, d, 2H, 2H, AA'BB'), 9.68 (s, 1H, CHSe); ${ }^{13} \mathrm{C}-\mathrm{NMR}\left(\mathrm{CDCl}_{3}\right): 63.62\left(\mathrm{CH}_{2} \mathrm{O}\right), 128.50$ (central benzene ring $\mathrm{Cq}$ ), 115.36 and 130.20 (side chain benzene $\mathrm{CH}$ ), 119.20, 152.40 (side chain benzene $\mathrm{Cq}$ ), $159.61(\underline{\mathrm{C}}=\mathrm{N}), 137.70$ ( $\underline{\mathrm{CHSe}})$; MS: $1500\left(\mathrm{M}^{+}\right.$, 100); Anal. \% Calcd. For $\mathrm{C}_{60} \mathrm{H}_{42} \mathrm{~N}_{12} \mathrm{O}_{6} \mathrm{Se}_{6}: \mathrm{C}, 48.01 ; \mathrm{H}, 2.82$; N, 11.20; Se, 31.57, Found: C, 47.91; H, $2.74 ; \mathrm{N}, 11.11$.

4-(4-\{2,4,5-Tri[4-(1,2,3-selenadiazole-4-yl)phenoxymethyl]benzyloxy\}phenyl)-1,2,3-selenadiazole (1b).

Yellow-orange solid (92\% yield); m.p. 87-88 C; IR: $v$ 3096, 2930, 1725, 1611, 1527, 1469, 1245, 1040, $822 \mathrm{~cm}^{-1}$; ${ }^{1} \mathrm{H}-\mathrm{NMR}(\mathrm{CDCl} 3): 5.25\left(\mathrm{~s}, 2 \mathrm{H}, \mathrm{CH}_{2} \mathrm{O}\right), 7.12,7.22\left(\mathrm{~d}, \mathrm{~d}, 2 \mathrm{H}, 2 \mathrm{H}, \mathrm{AA}^{\prime} \mathrm{BB}^{\prime}\right), 7.70$ (s, benzene central ring $\mathrm{CH}), 9.71(\mathrm{~s}, 1 \mathrm{H}, \mathrm{CHSe}) ;{ }^{13} \mathrm{C}-\mathrm{NMR}\left(\mathrm{CDCl}_{3}\right): 67.70\left(\underline{\mathrm{CH}}_{2} \mathrm{O}\right), 124.00$ (central benzene ring $\mathrm{CH}$ ), 129.80 (central benzene ring $\mathrm{Cq}$ ), 115.89 and 130.12 (side chain benzene $\mathrm{CH}$ ), 118.80, 152.60 (side chain benzene Ed) Calcd. For $\mathrm{C}_{42} \mathrm{H}_{30} \mathrm{~N}_{8} \mathrm{O}_{4} \mathrm{Se}_{4}$ : C, 49.13; H, 2.95; N, 10.91; Se, 30.77, Found: C, 49.10; H, 2.75; N, 10.71.

4-(4-\{3,5-Di[4-(1,2,3-selenadiazole-4-yl)phenoxymethyl]benzyloxy\} phenyl)-1,2,3-selenadiazole (1c).

Yellow-orange solid (87\% yield); m.p.100-102 ${ }^{\circ}$ C; IR: v 3096, 2917, 1738, 1610, 1534, 1450, 1239, 1046, $835 \mathrm{~cm}^{-1}$; ${ }^{1} \mathrm{H}-\mathrm{NMR}\left(\mathrm{CDCl}_{3}\right): 5.15$ (s, 2H, $\left.\mathrm{CH}_{2} \mathrm{O}\right), 7.11,7.20$ (d, d, 2H, 2H, AA'BB'), 7.49 (s, central benzene ring $\mathrm{CH}), 9.72(\mathrm{~s}, 1 \mathrm{H}, \mathrm{CHSe}) ;{ }^{13} \mathrm{C}-\mathrm{NMR}\left(\mathrm{CDCl}_{3}\right): 69.69\left(\mathrm{CH}_{2} \mathrm{O}\right), 126.00$ (central benzene ring $\mathrm{CH}$ ), 129.70 (central benzene ring $\mathrm{Cq}$ ), 115.99 and 130.03 (side chain benzene $\mathrm{CH}$ ), 118.80, 149.15 (side chain benzene $\mathrm{Cq}$ ), $160.15(\underline{\mathrm{C}}=\mathrm{N}), 137.60$ (대Se); MS: $789\left(\mathrm{M}^{+}, 100\right)$; Anal. \% Calcd. For $\mathrm{C}_{33} \mathrm{H}_{24} \mathrm{~N}_{6} \mathrm{O}_{3} \mathrm{Se}_{3}$ : C, 50.20; H, 3.06; N, 10.64; Se, 30.01, Found: C, 50.10; H, 3.00; N, 10.59 .

\section{1,4-Bis[4-(1,2,3-selenadiazol-4-yl)phenoxymethyl]benzene (1d).}

Yellow-orange solid (60\% yield); m.p. $160-162^{\circ} \mathrm{C}$; IR: v 3077, 2911, 1732, 1610, 1533, 1456, 1239, 1008, $822 \mathrm{~cm}^{-1}$; ${ }^{1} \mathrm{H}-\mathrm{NMR}$ (DMSO-d $\left.)_{6}\right) 5.15$ (s, 2H, $\mathrm{CH}_{2} \mathrm{O}$ ), 7.12, 7.21 (d, d, 2H, 2H, AA'BB'), 7.47 (d, benzene central ring $\mathrm{CH}), 9.72(\mathrm{~s}, 1 \mathrm{H}, \mathrm{CHSe}) ;{ }^{13} \mathrm{C}-\mathrm{NMR}\left(\mathrm{DMSO}-\mathrm{d}_{6}\right): 70.00\left(\underline{\mathrm{CH}}_{2} \mathrm{O}\right), 127.89$ (central benzene ring $\mathrm{CH}$ ), 129.18 (central benzene ring $\mathrm{Cq}$ ), 116.10 and 130.00 (side chain benzene $\mathrm{CH}$ ), 121.00, 152.00 (side chain benzene $\mathrm{Cq}$ ), $160.00(\underline{\mathrm{C}}=\mathrm{N}), 138.00$ (대Se); MS: $552\left(\mathrm{M}^{+}, 100\right)$; Anal. \% Calcd. For $\mathrm{C}_{24} \mathrm{H}_{18} \mathrm{~N}_{4} \mathrm{O}_{2} \mathrm{Se}_{2}$ : C, 52.18; H, 3.28; N, 10.14; Se, 28.60, Found: C, 52.03; H, 3.25; N, 10.20. 


\section{References}

1. Al-Smadi, M.; Meier, H. Liebigs Ann. 1997, 2357.

2. Al-Smadi, M.; Hanold, N.; Meier, H. J. Heterocycl. Chem. 1997, 34, 605.

3. Reddy, D. B.; Babu, N. C.; Padmavathi, V.; Padmaja, A. Tetrahedron 1997, 53, 17351.

4. Bhaskar Reddy, D.; Somasekhar Reddy, A.; Padmavathi, V. Synth. Commun. 2001, 31, 3429.

5. Lalezari, I.; Shafiee, A. Tetrahedron Lett. 1969, 5105.

6. Lalezari, I.; Shafiee, A. J. Org. Chem. 1971, 36, 2836.

7. Lalezari, I.; Shafiee, A.; Yalpani, M. J. Org. Chem. 1973, 38, 338.

8. Zhou, Y.; Heimgartner, H. Helv. Chem. Acta 2000, 83, 539.

9. Petrov, M. L.; Abramov, M. A.; Dehaen, W.; Toppet, S. Tetrahedron Lett. 1999, 40, 3903.

10. Detert, H.; Meier, H. Liebigs Ann. 1997, 1557.

11. Zhou, Y.; Heimgartner, H. Helv. Chem. Acta 2000, 83, 539.

12. Nishiyama, Y.; Hada, Y.; Anjiki, M.; Hanita, S.; Sonoda, N. Tetrahedron Lett. 1999, 40, 6293.

13. Arsenyan, P.; Oberte, K.; Pudova, O.; Lukevics, E. Chem. Heterocycl. Comp. 2002, 38, 1437.

14. Burling, F. T.; Goldenstein, B. M. J. Am. Chem.Soc. 1992, 114, 2313.

15. Jalilian, A. R.; Sattari, S.; Bineshmarvasti, M.; Daneshtalab, M.; Shafiee, A. Farmaco 2003, 58, 63.

16. Lalezari, I.; Shafiee, A.; Khorrami, J.; Soltani, A. J. Pharm. Sci. 1987, 67, 1336.

17. Abramov, M. A.; Dehaen, W.; D’Hooge, B.; Petrov, M. L.; Smeets, S.; Toppet, S.; Voets, M. Tetrahedron, 2000, 56, 3933.

18. D' Hooge, B.; Smeets, S.; Topren E.; Delarafm S. Fhem. Soc. Chem. Commun. 1997, 1753.

19. L'Abbe, G.; Haelterman, B.; Dehaen, W. J. Chem. Soc. Perkin Trans. 1 1994, 2203.

20. Malek-Yazdi, F.; Yalpani, M. Synthesis 1977, 328.

Sample availability: Contact the author.

(C) 2005 by MDPI (http://www.mdpi.org). Reproduction is permitted for noncommercial purposes. 